Relations industrielles

Industrial Relations

\title{
Contemporary Collective Bargaining in Seven Countries, par Adotf Sturmthal et d'autres, The Institute of International Industrial and Labor Relations, Cornell University, Ithaca, New York, 1957, 382 pp., \$4.50.
}

\section{Gaston Cholette}

Volume 14, numéro 3, juillet 1959

URI : https://id.erudit.org/iderudit/1022301ar

DOI : https://doi.org/10.7202/1022301ar

Aller au sommaire du numéro

\section{Éditeur(s)}

Département des relations industrielles de l’Université Laval

ISSN

0034-379X (imprimé)

1703-8138 (numérique)

Découvrir la revue

Citer ce compte rendu

Cholette, G. (1959). Compte rendu de [Contemporary Collective Bargaining in Seven Countries, par Adotf Sturmthal et d'autres, The Institute of International Industrial and Labor Relations, Cornell University, Ithaca, New York, 1957, 382 pp., \$4.50.] Relations industrielles / Industrial Relations, 14(3), 440-444.

https://doi.org/10.7202/1022301ar

Tous droits réservés (C Département des relations industrielles de l’Université Laval, 1959
Ce document est protégé par la loi sur le droit d'auteur. L'utilisation des services d'Érudit (y compris la reproduction) est assujettie à sa politique d'utilisation que vous pouvez consulter en ligne.

https://apropos.erudit.org/fr/usagers/politique-dutilisation/ 
le domaine social de 1871 à 1914. La première partie de ce travail est consacrée à une exposition des conditions de l'action sociale au cours de cetie époque. Dans les autres, l'auteur explore les différents champs d'activité: la famille, le travail urbain, le monde rural et maritime et enfin l'action auprès de l'Etat.

On suit le développement de groupements comme l'A.C.J.F., le Sillon, les Semaines sociales, l'Action populaire, les Secrétariats sociaux, le syndicalisme chez les employ'és, les organisations agricoles, etc. Des hommes, des équipes se forment et travaillent au milieu de difficultés fornłidables. Il est même surprenant qu'ils aient réussi à tenir le coup et à jeter les bases d'institutions qui ne pourront porter vraiment des fruits qu'après la première Grande guerre.

Cette époque a connu la crise du Modernisme, la Loi de Séparation, le Sillon, l'Accion française, etc. Les esprits conservateurs qui s'opposent à tout progrès avaient beau jeu pour utiliser l'opération classique de divension et camoufler leurs visées sociales et politiques derrière une prétendue orthodoxie qu'ils mettaient honteusement à leur service. Ils étaient d'ailleurs bien organisés. Quand on voit les attaques insidieuses des intégristes et les moyens dont ils disposaient dans les endroits stratégiques pour diffamer l'un après l'autre tous les groupements ou toutes les personnalités qui voulaient faire quelque chose dans le domaine social, on ne peut s'empêcher de songer qu'ils ont été sûrement parmi les plus grands responsables de l'abandon de l'Eglise par les masses populaires. Combien de fois, l'Action populaire, les Semaines sociales, les instigateurs du syndicalisme et d'oeuvres sociales ont perdu un temne précieux devant les Congrégations romaines pour s'expliquer, pour rectifier de faux rapports, pour se défendre. Il a fallu à tous une foi, un courage, une énergie indomptables pour réussir à passer à travers ces obstacles et continuer leur oeuvre avec l'approbation et le soutien de certains évêques clairvoyants.

Des ouvrages comme celui-ci rendent de très grands services. Ils nous permettent de constater comment le bien ne se fait pas tout seul. Ceux qui militent aujourd'hui dans le domaine social, comme tous ceux qui ont des responsabilités tireront grand avantage à con- naître cette histoire. Comme toute histoire bien faite, elle est une sagesse qui aide à apprécier le mouvement des idées, les hommes et les institutions dans une perspective plus large et dégagée de certaines contingences essentiellement caduques.

A cause du rôle important que joue la France au sein de l'Eglise et de l'influence qu'exercent les caiholiques sociaux de ce pays sur le Canada français, nous ne pouvons pas ignorer un ouvrage comme celui de M. Rollet. Même si ce n'était que pour nous metre en garde contre les difficultés et les embuches de cette époque - lesquelles, on le sait, ont toujours dhez nous leur effet à retardement - ce serait déjà beaucoup de leçons à apprendre.

\section{GÉrard Dion}

\section{En la Escuela de lo social. Carlos Giner et Dionisio Aranzadi, Instituto de estudios economico-sociales, Universi- dad de Deusto, 1958. 257 pp.}

Cet ouvrage n'a pas de prétention scientifique. C'est un manuel destiné à la formation sociale de la jeunesse. Les auteurs utilisent la méthode préconisée par la JOC: voir, juger, agir. Chaque chapitre est précédé d'un questionnaire bien fait. Ensuite, on procède de la manière suivante: un fait de la vie courante ou un exposé de doctrine est présenté au lecteur et ce n'est qu'après cela que l'on explique ce qu'en pense l'Eglise. Les auteurs connaissent bien tous les manuels d'enseignement social de l'Eglise, qui, pour la plupart, d'ailleurs, ont été traduits en espagnol. Ils sonit bien documentés, mais leur travail, qui est bien clair, bien divisé reste trop théorique, trop livresque Nous ne croyons pas qu'il fasse avancer la science, mais il semble proportionné au niveau des personnes auxquelles il est destiné et contribuera à créer chez eux une inquiétude salutaire.

GÉrard Dion

Contemporary Collective Bargaining in Seven Countries, par Adolf Sturmthal et d'autres, The Institute of International Industrial and Labor Relations, 
Cornell University, Ithaca, New York, 1957, 382 pp., $\$ 4.50$.

Les collaborateurs: Adolf STURMTHAL, professeur, Roosevelt University, Chicago; Allan FLANDERS, professeur, University of Oxford; John INMAN, éoonomiste, Colonial Office; P.S. PELS, secrétaire, Conseil économique et social, Pays-Bas; Clark KERR, chancelier. University of $\mathrm{Ca}$ lifornia; Luisa Riva SANSEVERINO, profeseur, Université de Pise; Cesare VANNUTELLI, professeur, Université de Rome; Neil CHAMBERLAIN, professeur, Columbia Univensity.

Voici un ouvrage très intéresant et extrêmement précieux pour tous ceux qui s'intéressent à la négociation collective. Il consiste en une série de sept études par des auteurs différents sur l'expérience de sept pays d'Europe et d'Amérique: la Grande-Bretagne, la Norvège, les Pays-Bas, la France l'Allemagne, l'Italie et les Etats-Unis. Le volume se termine par un essai sur la négociation collective comparée; cet essai est rédigé par Adolf Sturmthal, qui a joué le rôle d'animateur et de coordonnateur de l'oeuvre d'ensemble.

Bien que le cas de chaque pays soit traité par un auteur différent, les divers travaux comportent de grandes similitudes qui témoignent d'une communauté de dessein et qui donnent au volume une unité d'autant plus remarquable que son objet était une réalité fort complexe.

Les auteurs ont cherché à décrire la négociation collective et ses résultats dans le contexte historique et juridique des pays concernés. Ils font bien comprendre, en général, la structure particulière des parties intéressées à la négaciation, la nature et le degré de l'intervention gouvernementale dans les relations patronales-ouvrières, enfin et surtout l'un des principaux résultats de ces négociations, c'est-à-dire la structure des salaires.

L'étude sur la Grande-Bretagne a été préparée par Allan Flanders. L'auteur fait ressortir le degré exceptionnel de liberté et de variété qui caractérise l'expérience anglaise. Il analyse la structure des parties en présence, examine le mécanisme et le champ de la négociation, enfin traite de la structure et de la politique des salaires en insistant tout spécialement sur la période de plein emploi de l'après-guerre.

Allan Flanders conclut en soulignant la maturité des agents de la négociation collective en Grande-Bretagne. Mais cette maturité même, sans être assimilable à la stagnation, est un obstacle qui gêne l'évolution dynamique de la société industrielle.

Le cas de la Norvège est traité par J. Inman. Bien qu'il s'agisse avant tout d'une description et d'une analyse de la situation dans ce pays, cette étude prend surtout l'allure d'une thèse que l'auteur chercherait à faire valoir. Celuici s'attarde beaucoup moins que l'auteur précédent à la négociaiion collective elle-même; il s'étend au contraire sur les résultats de cette négociation.

Toutefois, Inman trace clairement le tableau de la structure des négociations. Il explique le caractère centralisé du mouvement patronal et du mouvement ouvrier, ce qui fait mieux comprendre la centralisation des négociations. On est en présence de deux forces qui se reconnaissent et s'acceptent pleinement, sous l'oeil vigilant d'un gouvernement de tendance ouvrière qui intègre les négociations dans l'ensemble de sa politique économique.

Les résultats des négociations sont groupés en deux périodies, celle qui va de 1945 à 1949 et ensuite celle de 1950 à 1955. La politique des salaires en Norvège a ceci d'unique qu'elle a été délibérément inspirée par un certain idéal de justice et qu'elle a effectivement abouti à un nivellement interindustriel et interprofessionnel. Dans la plupart des autres pays au contraire, notamment en Amérique du Nord, les salaires sont gradués selon le degré de profitabilité des branches d'activité économique. En Norvège, les ouvriers des secteurs privilégiés ont consenti à des sacrifices en faveur des groupes moins favorisés en échange contre une politique fiscale et une politique des prix qui faisaient partager le fardeau aux employeurs.

Pour les Pays-Bas, P.S. Pells présente un travail qui ressemble beaucoup, dans son plan, à celui qui précède. Il attache lui aussi plus d'importance et consacre un plus grand espace aux salaires qu'à la négociation. 
L'auteur parle d'abord des progrès de la négociation collective. Dans une deuxième section, il décrit les parties en présence et l'évolution institutionnelle de la collaboration patronale-ouvrière, principalement dans le champ de la négociation collective.

Il y a beaucoup de ressemblances entre la Norvège et les Pays-Bas: le mouvement ouvrier d'une pari, le monde patronal de l'autre, puis l'Etat intègrent leur action dans une politique d'ensemble qui a pour but d'établir une structure globale des salaires dans le pays sur la base de critères unanimement reconnus.

Le cas de la France est étudié par Adolf Sturmthal. L'auteur commence par l'hisıoire législative des négociations collectives, car la loi joue un rôle important dans un pays comme la France. Il étudie successivement les régimes établis par les lois de 1919, de 1936, de 1941, de 1946 et de 1950. Il souligne avec beaucoup de pertinence que la doctrine révolutionnaire qui a longtemps prévalu dans le syndicalisme ouvrier français a retardé le développement de la négociation collective; celleci en effet apparaissait comme une manoeuvre de diversion puisque la tâche essentielle était de combaitre politiquement le capitalisme. Il reste enoore beaucoup de traces de cet état d'esprit en France.

Sturmthal fait un bel exposé sur le mouvement ouvrier francais, sur la mentalité patronale et sur les lois qui fixent le régime des négociations. II fait bien ressortir la variété de la situation francaise, avec se notions d'associations les plus représentatives, d'extension des conventions aux tiers, avec la nature spéciale des négociations dans le secteur nationalisé et dans le cas des régies d'Etat.

Comme les autres auteurs, Surmthal accorde beaucoup d'espace au problème des salaires. Il parle du minimum vital interprofessionnel qui joue un si grand rôle en France, il insiste sur l'ampleur du salaire indirect que les Francais recoivent sous forme d'allocations familiales et d'autres paiements qui sont l'équivalent d'une redistribution du revenu national, enfin il situe bien le rôle considérable du gouvernement dans la politique générale des salaires. Son étude est probablement la meilleure de celles. qui viennent d'être commentées.

Le clou du volume, c'est à mon avis le magnifique travail de Clark Kerr sur l'Allemagne d'après-guerre. Il est difficile d'imaginer une analyse plus lucide et plus pénétrante de la structure des parties en présence, des forces centrifuges et centripètes qui agissent en chacune d'elles, des mécanismes de la négociation, des influences qui contribuent à faire évoluer les relaiions patronalesouvrières dans un sens plutôt que dans l'autre.

Clark Kerr accorde proportionnellement moins d'importance que les autres auteurs aux salaires et consacre beaucoup plus de temps à la description de la négociation collective. Il met en lumière la structure quasi corporative de l'organisation patronale-ouvrière en Allemagne. $\mathrm{Ce}$ ne sont pas les entreprises mais les associations patronales qui comptent dans ce pays; non seulement ces groupements négocient-ils, mais ils exercent une sorte de règlementation du commerce et de l'industrie. Du côté ouvrier, même phénomène de centralisation et d'absence de vitalité à la base, sur le plan local.

L'auteur étudie les espèces de conventions et leur contenu. Il montre l'importance croissante des tribunaux du travail institués par l'Etat. Il traite du système d'extension des conventions collectives qui est presque entièrement semblable au régime existant dans la province de Québec. Ses commentaires sur l'impact de ce régime sur le syndicalisme patronal et ouvrier sont très pertinents et ingénieux.

En conclusion, Clark Kerr constate la prédominance des facteurs religieux et politique sur les motivations d'ordre économique en Allemagne et il met en lumière le besoin de dirigisme et de sécurité chez le peuple allemand.

Il y a deux études sur l'Italie. La première, par Luisa Riva Sanseverino, n'est pas du même calibre que les autres de ce volume. L'auteur s'attarde sur les salaires et ses divers éléments. Les renseignements sur la négociation collective ne sont pas satisfaisants et il y a de la confusion au sujet de l'intervention gouvernementale. On ne voit pas bien les forces qui sont à l'oeuvre et on 
ne parvient pas à localiser les centres de décision.

Les négociaions se font dans une bonne mesure sur le plan national. Sur le plan local il y a des commissions qui ressemblent aux comités mixtes de produotion canadiens et aux comités d'entreprise français mais qui sont en rivalité avec les syndicats ouvriers à cause de leur juridiction concurrente.

Un des éléments typiques de la situation en Italie, c'est la limitation rigide du droit de licenciement de l'employeur. Les congédiements pour raisons disciplinaires relèvent des tribunaux ordinaires; les mises-à-pied sont soumises à l'arbitrage.

La deuxième étude sur l'Italie porte exclusivement sur la structure des salaires et le coût de la main-d'oeuvre. Cesare Vannutelli traite sucoessivement du salaire de base, de l'indemnité relative au coût de la vie, de l'allocation pour le pain, puis des réajuscements entre les catégories de travailleurs selon la compétence. Il aborde ensuite d'autres éléments de la rémunération ouvrière, qui sont des réalisations typiquement italiennes, par exemple tous les congés statutaires qui ne tombent pas un dimanche, le cadeau de Noël et la paye de licenciement. Enfin l'auteur étudie les paiements faits en vertu de lois de sécurité sociale.

Il aurait été préférable qu'une seule étude soit faite sur l'Italie. De cette façon, il n'y aurait probablement pas eu de disproportion aussi marquée entre l'importance accordée à la négociation collective et celle qui est attribuée au problème des salaires. Cette dernière question occupe toute la place dans le travail de Vannutelli et beaucoup trop d'espace dans celui de Sanseverino.

L'étude sur les Etats-Unis a été faite par Neil W. Chamberlain. C'est une oeuvre remarquable, comme la plupart de celles que cet auteur a écrites.

Au début, Chamberlain fait de la géographie syndicale; il situe le mouvement ouvrier dans le contexte industriel des Etats-Unis. Il en trace l'évolution historique en soulignant les grands points de repère: l'adoption du National Labor Relations Act, la formation du CIO, enfin l'amalgamation des deux grandes centrales ouvrières.
L'auieur parle ensuite du champ de négociation de la convention collective puis, sous le titre de « The Pattern of Bargaining », il analyse et décrit à merveille le mécanisme de la négociation. Cette partie de son étude est la meilleure, ce qui n'est pas peu dire. Pour illustrer ses explications, lauteur donne ensuite un aperçu général des principales négociations-clés aux Etats-Unis après la guerre en $\mathrm{y}$ ajoutant une description de la marche de leurs répercussions à travers l'économie nord-américaine.

La deuxième partie du travail de Chamberlain porte sur les salaires. Elle est moins forte que la première, en ce sens qu'elle est moins cohérente et qu'elle fait plus de place à des emprunts à d'autres auteurs qu'à la pensée propre de Chamberlain. Celui-ci analyse l'influence à court terme et à longue échéance de l'action syndicale sur les salaires. Il se demande ensuice selon quels critères devrait s'édifier une théorie sur une saine politique nationale des salaires. Après ces considérations, l'auteur traite du problème des grèves en temps de paix, de la question des grèves nationales, enfin de la politique des salaires et du contrôle des grèves en temps de crise. Cette matière ne se trouve que dans l'étude de Chamberlain: les autres auteurs ne l'ont même pas abordée.

Le travail de Chamberlain contient enfin un très bref aperçu sur la matière des conventions collectives et des remarques fort originales sur la procédure de règlement de griefs dont le fonctionnement offre d'excellentes chances de promotion humaine à l'échelle locale.

Enfin le volume se termine par un essai d'Adolf Sturmthat sur la négociation collective comparée. Cet essai n'a pas pour but de comparer les systèmes de négociation collective entre les divers pays, mais plutôt d'utiliser l'expérience de chacun de ces pays dans l'analyse de certaines questions choisies qui se rattachent à la négociation collective: l'importance relative de la négociation comme mécanisme de fixation des salaires et des conditions de travail, la rationalisation et la centralisation des négociations, l'extension des conventions collectives, la structure des salaires dans la période d'après-guerre, enfin la politique 
des salaires dans l'hypothèse du plein emploi.

Plus de la moitié de l'étude de Sturmthal porte sur les salaires; le reste a pour objet la négociation. Au début l'auteur signale la difficulté de comparer le degré d'importance de la négociation d'un pays à l'autre. Lorsqu'il traite de la centralisation des négociations et de l'uniformisation conséquente des conditions de travail, Sturmthal est très intéressant, mais il excelle quand il analyse le problème de l'extention légale des conventions et il ne manque pas alors de scruter avec beaucoup de pénétration la psychologie patronale et ouvrière afin d'expliquer les causes et les effets de ce phénomène.

Dans la partie qui se rapporte aux salaires, Sturmthal commence par comparer les différences entre les taux des ouvriers qualifiés et ceux des travailleurs qui ne le sont pas. Il constate que la différence de niveau entre ces deux catégories est beaucoup plus restreinte en Europe qu'aux Etats-Unis. Il fait sur ce point l'inventaire de plusieurs hypothèses mais ne trouve pas d'explication satisfaisante. Il ne consacre même pas une page entière par la suite aux différences entre les branches d'activité écoromique, entre les salaires des hommes et des femmes, et à peine plus aux différences régionales.

L'auteur s'arrête brièvement au problème d'une structure «rationnelle 》 des salaires, et signale que là où de sérieux efforts ont été accomplis dans cette direction, la réalité ne s'est pas laissée plier aux cadres rigides qu'on a cherché à lui imposer. Il traite assez longuement de la négociation collective et des niveaux de salaires. La négociation a tendance à uniformiser les salaires, mais le niveau qui sert de barême n'est pas le même dans tous les régimes: aux Etats-Unis, par le truchement des négociations-clés (pattern bargaining ) les salaires ont tendance à s'aligner au niveau de ceux des branches d'activité et des entreprises les plus prospères; en Europe, au contraire, ils sont liés au niveau de la capacité de payer des entreprises marginales. Cette méthode européenne réduit la concurrence et facilite la transformation de l'augmentation des salaires en des majorations de prix dans tout le marché.

Sturmthal termine son essai par des réflexions sur le problème de la stab1- lité des prix dans une économie de plein emploi. Il conclut en affirmant qu'il n'existe aucune méthode qui soit également efficace d'un pays à l'autre dans la recherche de cet équilibre.

\section{Gaston Cholette}

Les doctrines économiques, par A. Hamelin. Collection «Vous connaîtrez 》. Les Editions ouvrières, 12, avenue Soeur-Rosalie, Paris, 13. 1959, 186 pages.

L'auteur présente une synthèse des différentes doctrines économiques préconisées à travers les âges. Il s'attache particulièrement au mercantilisme, aux physiocrates, à l'école libérale ou classique, au socialisme et aux écoles nonsocialisies. Enfin, il dit un mot de la réaction contre la science classique: doctrines historique et hédoniste. Malheureusement, il ne s'étend pas assez sur les doctrines de l'époque contemporaine, celles qui nous touchent plus profondément. C'est un ouvrage de vulgarisation pour les gens pressés qui n'ont pas le temps d'étudier à fond les ouvrages spécialisés. Cependant nous craignons qu'en raison de la lacune signalée, ils n'y trouvent pas tout $\infty$ qu'ils attendent.

GÉrard Dion

Forces et faiblesses des entreprises familiales. Centre d'études des problèmes industriels. Edition «Pilote 》, 109, rue de la Bassée, Lille, 1959, 157 pp.

La plupart des études en relations du travail portent sur les entreprises de grandes dimensions, organisées en corporations et dirigées par un bureau de direction représentant juridiquement des actionnaires anonymes. S'il est vrai que notre économie est marquée par ce genre d'entreprises à cause de leur importance économique et de la quantité de main-d'oeuvre qu'elles emploient, il est incontestable qu'au Canada, comme aux Etats-Unis et dans la plupart des pays d'Europe occidentale, la forme familiale de l'entreprise est encore celle qui est la plus répandue. Et le phénomène de concentration industrielle ne la fera pas disparaître, loin de là; elle aura toujours sa place parce qu'elle exerce une 